\title{
Diagnosis of Vulvovaginal Candidiasis and Effectiveness of Combined Topical Treatment with Nystatin: Results of a Non-Interventional Study in 973 Patients
}

\author{
G. Dressen ${ }^{\#, 1}$, W. Kusche $e^{\#, 2}$, C, Neumeister ${ }^{*, 3}$ and U. Schwantes ${ }^{\#, 3}$ \\ ${ }^{1}$ Gynaecological Surgery, Eisenbahnstrasse 27, D-66117 Saarbruecken, Germany \\ ${ }^{2}$ A.CRO Clinical Research Services GmbH, D-65191 Wiesbaden, Germany \\ ${ }^{3}$ Department of Medical Science/Clinical Research, Dr. R. Pfleger GmbH, D-96045 Bamberg, Germany
}

\begin{abstract}
Introduction \& Objectives: This non-interventional study was performed in 231 gynaecological surgeries in order to assess current routine diagnostic practice as well as present effectiveness and tolerability of a combined topical treatment with nystatin in patients with uncomplicated vulvovaginal candidiasis (VVC).

Methods: Naïve or insufficiently treated VVC patients received a combined topical antimycotic treatment ${ }^{\S}$. Diagnostic procedures were documented. Cardinal disease symptoms and dosage regimens were recorded before and after therapy. Investigators and patients rated treatment effectiveness and tolerance. Adverse events and withdrawals were documented.

Results: All 1,011case reports were included in the safety analysis; thereof 973 cases in the efficacy analysis. Based on diagnoses conform to guidelines $74.7 \%$ of the patients had a primary infection while $24.9 \%$ were experiencing a relapse. After a treatment period of 6 days (median), individual symptoms had ceased in up to 81.9\%. Complete healing was achieved in $63.1 \%$ (vulva) and $65.4 \%$ (vagina). Investigators rated therapy outcome as "healing/significant improvement" in $90.0 \%$ (vulva) and $88.2 \%$ (vagina), and tolerance "as very good/good" in $97.4 \%$.

Conclusions: Routine diagnosis of VVC predominantly corresponds to relevant guidelines. Combined intra- and perivaginal therapy of VVC with nystatin is an effective, safe and cost-conscious treatment option. Nystatin has not lost its effectiveness over the years.

${ }^{\S}$ Nystatin cream and vaginal tablets (Biofanal ${ }^{\circledR} 100.000$ IU combination package), Dr. R. Pfleger GmbH, D-96045 Bamberg, Germany
\end{abstract}

Keywords: Vulvovaginal candidiasis, diagnosis, nystatin, combined treatment, vaginal tablets and cream.

\section{INTRODUCTION}

Nystatin, discovered in 1948, was introduced as the first effective antimycotic treatment in the 1950s [1]. Since then, nystatin has been used worldwide to date for the cure of various superficial and intestinal Candida infections in all age groups $[2,3]$. In contrast to some azole agents no therapeutically relevant impairment of antimycotic efficacy against the most relevant species in vulvovaginal candidiasis (VVC) has been observed over the years [4-8].

National guidelines on diagnosis and treatment of VVC recommend microscopy and culture to confirm diagnosis in uncomplicated VVC beside clinical and colposcopic examination $[9,10]$.

This study was performed to investigate current routine diagnostic procedures as well as present efficacy and

*Address correspondence to this author at the Department of Medical Science/Clinical Research, Dr. R. Pfleger GmbH, D-96045 Bamberg, Germany; Tel: +49 9516043 161; Fax: +49 9516043 226;

E-mail: Claudia.Neumeister@dr-pfleger.de

${ }^{\#}$ These authors contributed equally to this work. tolerability of combined nystatin treatment in daily gynaecological practice. Moreover, results of a small-sized noninterventional study involving concomitant use of nystatin cream and vaginal tablets for the treatment of VVC should be verified in a large number of patients. The preceding study was requested by the federal regulatory authority in Germany (BfArM) for re-registration purposes.

\section{MATERIALS AND METHODOLOGY}

1,011 patients were enrolled in this non-interventional study at 231 gynaecologic surgeries in Germany. Patients received an information sheet and signed a consent form regarding data protection. Inclusion criteria were the presence of VVC symptoms and the indication for a local therapy with nystatin cream and vaginal tablets. Gynaecologists were questioned as to whether they used clinical inspection, colposcopy, microscopic examination of vaginal secretions, and culture to ensure correct VVC-diagnosis. With regard to dosing and treatment duration, no specific specifications were made, but investigators were advised to adhere to the instructions in the package leaflet.

The individual case report forms required collection of the following data at visit 1: demography, anamnestic and 
current clinical findings and symptoms of candidosis, predisposing factors and respective medical pre-treatment, state of a possible pregnancy, and prescription details of nystatin. 7 to 10 days later on visit 2 , the following was to be recorded: administration details of nystatin, current symptoms, investigators' and patients' assessment of effectiveness and tolerance, and any adverse drug effects.

Data were entered into an electronic data base and descriptive statistics (absolute and relative frequency, mean, standard error of the mean, median, standard deviation, variance, minimum, maximum, percentiles) were carried out by using the SPSS 10 statistical software. Validation of data was checked within the data base and by comparing $5 \%$ of data fed into the data base with those in the related data sheets.

The observational study protocol was submitted to an ethics committee (freiburger ethic-kommission $\mathrm{GmbH}$ international, Freiburg, Germany) prior to study start. The ethical committee approved the study.

In compliance with German drug law, competent authorities were given notice of the study and the official recommendations for the conduct of non-interventional studies were observed [11].

\section{RESULTS}

\section{Study Population and Basic Demographic Data}

Of the 1,011 case documentations obtained, data from 38 patients were excluded from analysis due to violations of the observational study protocol (retrospective documentation or single application of either cream or vaginal tablets), which rendered 973 valid data sets for evaluation including 200 pregnant patients.
The mean age of the patients was $34.8 \pm 12.9$ years (median 32, min. 15, max. 85) and mean weight was $68.1 \mathrm{~kg}$ (median 66kg, min. 40kg, max. $185 \mathrm{~kg}$ ).

\section{History and Severity of Vulvovaginal Symptoms at Baseline}

At visit 1, patients had a mean history of vulvo-vaginal symptoms of $5.9 \pm 5.8$ days (median 4 , min. 0, max. 90). 727 patients $(74.4 \%)$ experienced these symptoms for the first time; 243 patients $(24.9 \%)$ suffered from a recurrent infection after a mean symptom free period of 5.7 months. In $57.9 \%$ of the patients, vulvitis was diffuse-eczematous, in $20.4 \%$ vesiculous-pustulous, and in $8.0 \%$ follicular. In $48.6 \%$ of the patients the outer infection was limited to the labia, but in $40.2 \%$ neighbouring regions were also affected. In the majority of patients $(57.9 \%)$ a medium colpitis was diagnosed, minor or severe manifestations were less frequent. At study start respective vulvovaginal symptoms (itching, vaginal discharge and burning pain) were present in $83.8 \%$ to $95.3 \%$ of the patients (Table 1a).

In addition to the clinical inspection VVC-diagnosis was confirmed by colposcopy in $60.5 \%(n=589)$ of patients. Microscopic examination of vaginal secretions was carried out in $86.9 \%(n=864)$ and culture tests in $22.8 \%(n=222)$ of cases. Candida (predominantly Candida albicans, in single cases Candida glabrata and Candida tropicalis) was identified as the pathogen in $81.5 \%$ of all culture tests.

The most frequent predisposing factors for the infection were hormonal contraception $(35.3 \%)$, pregnancy $(20.6 \%)$ and systemic antibiotics $(12.1 \%)$, followed by adiposity $(7.5 \%)$, seniority $(5.3 \%)$ and climacteric period $(5.2 \%)$.

Table 1. Vulvovaginal Symptoms Before and After Treatment a. Severity of vulvovaginal symptoms before therapy with nystatin cream and tablets $(n=973)$

\begin{tabular}{|c|c|c|c|c|c|c|c|c|c|c|}
\hline \multirow{3}{*}{ Symptom } & \multicolumn{10}{|c|}{ Severity of vulvovaginal symptoms before treatment } \\
\hline & \multicolumn{2}{|c|}{ severe } & \multicolumn{2}{|c|}{ moderate } & \multicolumn{2}{|c|}{ minor } & \multicolumn{2}{|c|}{ absent } & \multicolumn{2}{|c|}{ no data } \\
\hline & $\mathbf{n}$ & $\%$ & $\mathbf{n}$ & $\%$ & $\mathbf{n}$ & $\%$ & $\mathbf{n}$ & $\%$ & $\mathbf{n}$ & $\%$ \\
\hline Itching* & 256 & 26.3 & 481 & 49.4 & 190 & 19.5 & 42 & 4.3 & 4 & 0.4 \\
\hline Burning* & 118 & 12.1 & 414 & 42.5 & 283 & 29.1 & 151 & 15.5 & 7 & 0.7 \\
\hline Discharge & 148 & 15.2 & 481 & 49.4 & 274 & 28.2 & 67 & 6.9 & 3 & 0.3 \\
\hline
\end{tabular}

b. Severity of Vulvovaginal Symptoms After Therapy with Nystatin Cream and Tablets (n=966)

\begin{tabular}{|c|c|c|c|c|c|c|c|c|c|c|}
\hline \multirow{2}{*}{ Symptom } & \multicolumn{10}{|c|}{ Severity of vulvovaginal symptoms after treatment } \\
\hline & $\mathbf{n}$ & $\%$ & $\mathbf{n}$ & $\%$ & $\mathbf{n}$ & $\%$ & $\mathbf{n}$ & $\%$ & $\mathbf{n}$ & $\%$ \\
\hline Itching* & 4 & 0.4 & 36 & 3.7 & 234 & 24.2 & 691 & 71.5 & 1 & 0.1 \\
\hline Burning & 2 & 0.2 & 24 & 2.5 & 141 & 14.6 & 791 & 81.9 & 8 & 0.8 \\
\hline
\end{tabular}

$*$ Sum $\neq 100 \%$ due to rounding differences. 


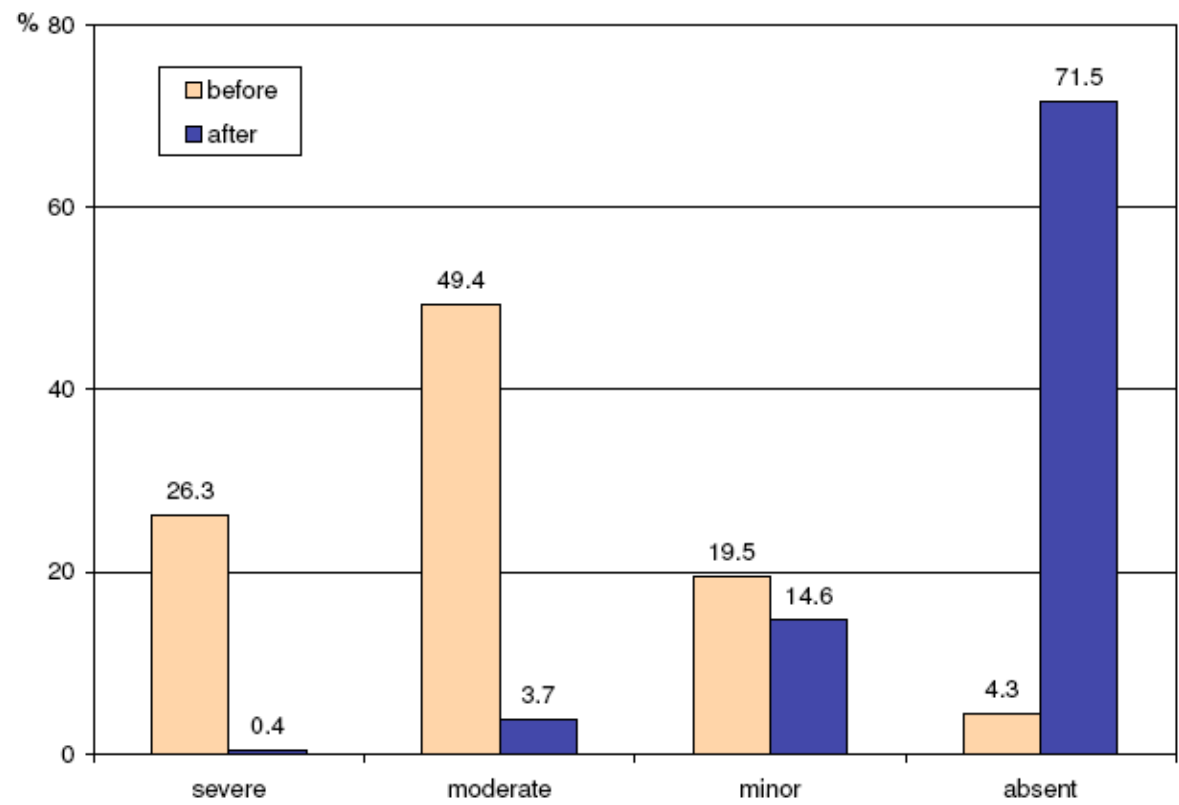

Fig. (1). Symptom "itching" before and after local combination treatment with nystatin.

\section{Indication Specific Therapy Before Study Entry}

At study entry, $93.4 \%$ of 243 patients with a recurrent infection had already received pre-treatment which was mostly local $(86.6 \%)$. Of the 727 patients with a primary infection, only $5.2 \%$ were pre-treated.

Imidazole derivatives (63.4\%), nystatin $(6.2 \%)$ and ciclopiroxolamin $(5.1 \%)$ were the most frequently applied topicals. In $95.6 \%$ of the cases, lacking efficacy or recurrent symptoms were the reason for the therapy change.

\section{Therapy Regimens in this Study}

Patients were instructed to use nystatin vaginal tablets and cream for a median period of 6 days. The most frequently prescribed doses were $1(68.8 \%)$ or $2(25.9 \%)$ vaginal nystatin tablets daily. In addition, patients were asked to apply nystatin cream twice a day (66.3\%), three times a day $(12.9 \%)$ or only once a day $(18.1 \%)$. Treatment compliance was critically examined for both formulations at visit 2 and showed extensive compliance.

\section{Vulvovaginal Symptoms After Treatment}

After combination treatment with nystatin vaginal tablets and cream, a clear reduction of all vulvovaginal symptoms was evident in the 966 patients completing visit 2 (Table 1b). The fraction of patients without itching was now $71.5 \%$ (previously 4.3\%; Fig. 1), without burning $81.9 \%$ (previously $15.5 \%$ ), and without vaginal discharge $64.9 \%$ (previously $6.9 \%$ ). Furthermore, of the 758 patients with all of the aforementioned symptoms at visit 1, 351 patients (46.3\%) were free of any symptom after treatment.

\section{Investigators' and Patients' Rating of Treatment Efficacy}

The general assessment of treatment efficacy by investigators (differentiated vulva/vagina) and patients is shown in Table 2. In up to $90.0 \%$ of the cases, the investigators rated the therapy outcome as "healing" or "significant improvement" and only in $3.0 \%$ as "no change" or "worsening". The patients' ratings were widely comparable.

Table 2. Investigators' Rating of Treatment Efficacy

\begin{tabular}{|c|c|c|c|c|}
\hline \multicolumn{2}{|c|}{ Vulva } & \multicolumn{2}{c|}{ Vagina } \\
\hline & N & $\%$ & n & $\%$ \\
\hline \hline Healing & 910 & 63.1 & 632 & 65.4 \\
\hline Significant improvement & 260 & 26.9 & 220 & 22.8 \\
\hline Moderate & 44 & 4.6 & 45 & 4.7 \\
\hline No change & 23 & 2.4 & 21 & 2.2 \\
\hline Worsening & 1 & 0.1 & 2 & 0.2 \\
\hline No data & 28 & 2.9 & 45 & 4.8 \\
\hline Total & 966 & 100 & 966 & 100 \\
\hline
\end{tabular}

Treatment efficacy as judged by investigators $(n=966)$.

\section{Treatment Tolerance}

General tolerance of local nystatin treatment was rated as "very good" or "good" by $97.4 \%$ of the investigators and $95.5 \%$ of the patients. $2.5 \%$ of the patients judged the treatment tolerance as "moderate", another $0.6 \%$ as "poor ".

\section{Therapy Withdrawals and Side Effects}

Treatment with nystatin was prematurely terminated in 11 cases $(1.1 \%)$ : 3 due to lack of efficacy, 2 due to lacking acceptance by the patient, 2 irrespective of the treatment, 1 due to a feeling of dryness, 1 due to freedom from symptoms.

Two additional withdrawals were treated as potential side effects. One patient suffered from local reactions and 1 preg- 
nant patient reported the temporary on-set of contractions. Another patient suffered from local reactions but did not terminate treatment prematurely. In these 3 patients the complaints disappeared. In the case of the pregnant patient, childbirth took place at the expected time (week $40+2$ of pregnancy).

\section{DISCUSSION}

Results of this non-interventional study indicate that routine diagnosis of uncomplicated VVC in German gynaecological surgeries is predominantly carried out in agreement with the procedures recommended in relevant guidelines [9, 10]. Mean age of the patients was 34.8 , which corresponds to the occurrence peak of VVC in the third decade of life [3].

Since Azole therapy seems to be unreliable for nonalbicans species [3, 12, 13], nystatin continues to be a valuable agent in the treatment of vulvovaginal Candida infections [7, 12]. The healing rates obtained with nystatin cream and vaginal tablets in patients with VVC are comparable with data reported over the years [7, 14, 15, 16], also confirming that resistance of Candida to nystatin has still not become an issue of concern in gynaecological practice.

The outcome of this investigation is in agreement with the results of the preceding small-sized study and supported by results of a recent clinical trial in complicated vulvovaginal candidosis patients published in 2010 [7]. The authors report practically identical mycological cure rates of $85.6 \%$ for fluconazole and $85.4 \%$ for nystatin after $7-14$ days.

Due to its efficacy and low risk profile, nystatin remains the first line treatment for Candida infections in the first pregnancy trimester [17]. Although other potent antimycotic agents have been developed over the years, the therapeutic value of nystatin is unquestionable. Recent investigations demonstrate comparable or higher susceptibility of Candida species for topical nystatin compared to clotrimazole, itraconazole, fluconazole, miconazole or terbinafine [8, 18-21]. While resistance for azoles such as fluconazole and econazole plays an increasing role in the treatment of candida vulvovaginitis $[22,23]$, this seems not to be the case for nystatin $[8,18,21,23]$ Nystatin remains an efficient, safe and economic option in the treatment of VVC infections.

\section{CONCLUSIONS}

Combined local treatment of vulvovaginal candidiasis with nystatin cream and vaginal tablets was as effective and safe under routine conditions in gynaecological practice as demonstrated earlier in controlled clinical trials.

\section{CONFLICT OF INTEREST}

Dressen $\mathrm{G}$ - Consultant of the Sponsor

Kusche W - Employee of A.CRO GmbH, the contract research organization commissioned with data management and analysis

Neumeister C and Schwantes U - Employees of the Sponsor

\section{ACKNOWLEDGEMENTS}

The study was funded by the manufacturer Dr. R. Pfleger $\mathrm{GmbH}$, Bamberg, Germany.

\section{Responsibilities}

German Dressen contributed in the study and was involved in preparing the manuscript. Claudia Neumeister was responsible for the study design and project management. Ulrich Schwantes was responsible for supervision of the study and editing the manuscript. Werner Kusche was responsible for trial data evaluation and reporting and for drafting the manuscript.

The authors wish to thank all other contributing gynaecologists in Germany for their valuable cooperation. We thank Anna Wolf for proofreading the manuscript.

\section{REFERENCES}

[1] Baldwin RS. The Fungus Fighters: Two Women Scientists and Their Discovery, Cornell University Press, Ithaka and London 1981.

[2] Fölster-Holst R, Höger P. Pustular diseases of the newborn. J Dtsch Dermatol Ges 2004; 2(7): 569-79.

[3] Sobel JD. Vulvovaginal candidosis. Lancet 2007; 369: 1961.

[4] Baier R, Puppel H. Antimycotic sensitivity of yeasts from clinical specimens. Dtsch med Wochenschr 1978; 103(27): 1112-6.

[5] Hudson MMT. Antifungal resistance and over-the-counter availability in the UK: a current perspective. J Antimicrob Chemother 2001; 48(3): 345-50.

[6] Richter S, Galask RP, Messer SA, Hollis RJ, Diekema DJ, Pfaller MA. Antifungal Susceptibilities of Candida Species Causing Vulvovaginitis and Epidemiology of Recurrent Cases. J Clin Microbiol 2005; 43(5): 2155-62.

[7] Fan SR, Liu XP. In vitro fluconazole and nystatin susceptibility and clinical outcome in complicated vulvovaginal candidosis. Mycoses no. Available from: http://onlinelibrary.wiley.com/doi/ 10.1111/j.1439-0507.2010.01887.x/full. Accessed on: 2010 Apr 6.

[8] Vráblik J, Masata J, Jedlicková A, Hájíckova M. Prospective study the prevalence of different candida strains and their sensitivity to different antimycotic treatment in women with vulvovaginal candidiasis. Ceska Gynekol 2007; 72(1): 27-32.

[9] White DJ, Vanthuyne A. Vulvovaginal candidiasis (VVC). In: Ross J, Ison C, Carder C, Lewis D, Mercey D, Young H. Sexually transmitted infections: UK national screening and testing guidelines. London, British Association for Sexual Health and HIV (BASHH), 2006; 68-75.

[10] British Association for Sexual Health and HIV, Clinical effectiveness group 2007: UK National guideline on the management of vulvovaginal candidiasis. Available from http:/www.bashh.org

[11] Empfehlungen zur Planung, Durchführung und Auswertung von Anwendungsbeobachtungen. German Fed Gaz 1998; 229, Accessed on: 04.Dec.1998.

[12] Rex JH, Walsh TJ, Dobel JD, et al. Practice Guidelines for the Treatment of Candidiasis. Clin Infect Dis 2000; 30: 662-78.

[13] Sojakova M, Liptajova D, Borovky M, Subik J. Fluconazole and itraconazole susceptibility of vaginal yeast isolates from Slovakia. Mycopathologia 2004; 157(2): 163-9.

[14] Konnegen V, Richter P, Blaschke-Hellmessen R. Effectiveness of nystatin $\mathrm{G}$ in the treatment of vaginal mycoses. Zentralblatt für Gynäkologie 1980; 102(1): 49-52.

[15] Arikan S, Ostrosky-Zeichner L, Lozano-Chiu M, et al. In vitro Activity of Nystatin Compared with Those of Liposomal Nystatin, Amphotericin B, and Fluconazole against Candida Isolates. J Clin Microb 2002; 40(4): 1406-12.

[16] Göttlicher S. Kurzzeitbehandlung der akuten vulvovaginalen Mykose mit Nystatin - Eine prospektive Studie an 150 Patientinnen. Therapiewoche 1989; 39(20): 1450.

[17] Schaefer C, Spielmann H, Vetter K. Arzneiverordnung in Schwangerschaft und Stillzeit. 7th ed. Munich, Urban \& Fischer Verlag, Elsevier, 2006. 
[18] Huang YY, Deng MD, Lai YR. et al. Species of candida and drug susceptibility of recurrent vulvovaginal candidiasis. Matern Child Health Care Chin 2009; 5. Available from: http://en.cnki.com.cn/ Article_en/CJFDTOTAL-ZFYB200905031.htm

[19] Akah PA, Nnamani CE, Nnamani PO. Prevalence and treatment outcome of vulvovaginal candidiasis in pregnancy in a rural community in Enugu State, Nigeria. J Med Med Sci 2010; 1(10): 447-52.

[20] Martins HPR, Da Silva MC, Paiva LCF, Svidzinski TIE, Consolaro MEL. Efficacy of Fluconazole and nystatin in the treatment of vaginal Candida species. Acta Derm Venereol 2012; 92: 78-82.
[21] Yu F, Zhou Y, He L. The etiologic character of recurrent vulvovaginal candidiasis and drug sensitivity analysis. Chin J Mod Med 2007; 23. Available from http://en.cnki.com.cn/Article_en/ CJFDTOTAL-ZXDY200723032.htm

[22] Belliti F, Cuniato V, Nocera E, et al. Candida vulvovaginitis: an epidemiological survey among immigrant prostitutes. Infez Med 2002; 10(1): 31-6.

[23] Asticcioli S, Sacco L, Daturi R et al. Trends in frequency and in vitro antifungal susceptibility patterns of candida isolates from women attending the STD outpatients clinic of a tertiary care hospital in Northern Italy during the years 2002-2007. New Microbiologica 2009; 32: 199-204.

(C) Dressen et al.; Licensee Bentham Open.

This is an open access article licensed under the terms of the Creative Commons Attribution Non-Commercial License (http://creativecommons.org/ licenses/by-nc/3.0/) which permits unrestricted, non-commercial use, distribution and reproduction in any medium, provided the work is properly cited. 